PROCEEDINGS OF THE

AMERICAN MATHEMATICAL SOCIETY

Volume 131, Number 3, Pages 911-920

S 0002-9939(02)06578-4

Article electronically published on July 17, 2002

\title{
OPERATOR KERNEL ESTIMATES FOR FUNCTIONS OF GENERALIZED SCHRÖDINGER OPERATORS
}

\author{
FRANÇOIS GERMINET AND ABEL KLEIN
}

(Communicated by Joseph A. Ball)

\begin{abstract}
We study the decay at large distances of operator kernels of functions of generalized Schrödinger operators, a class of semibounded second order partial differential operators of mathematical physics, which includes the Schrödinger operator, the magnetic Schrödinger operator, and the classical wave operators (i.e., acoustic operator, Maxwell operator, and other second order partial differential operators associated with classical wave equations). We derive an improved Combes-Thomas estimate, obtaining an explicit lower bound on the rate of exponential decay of the operator kernel of the resolvent. We prove that for slowly decreasing smooth functions the operator kernels decay faster than any polynomial.
\end{abstract}

\section{INTRODUCTION}

In this article we establish decay estimates on operator kernels of functions of generalized Schrödinger operators, a class of semibounded second order partial differential operators of mathematical physics, which includes the Schrödinger operator, magnetic Schrödinger operator, and the classical wave operators (i.e., acoustic operator, Maxwell operator, and other second order partial differential operators associated with classical wave equations).

We fix the dimension $d$. For each $k=1,2, \ldots$ we set

$$
\mathcal{H}^{(k)}=\mathrm{L}^{2}\left(\mathbb{R}^{d}, \mathrm{~d} x ; \mathbb{C}^{k}\right) .
$$

The spectrum and the domain of an operator $B$ will be denoted by $\sigma(B)$ and $\mathcal{D}(B)$, respectively. If $B$ is a semibounded operator we will denote the corresponding quadratic form by $B[\psi]$, with $\psi \in \mathcal{Q}(B)$, its form domain. $(B[\psi]=\langle\psi, B \psi\rangle$ for $\psi \in \mathcal{D}(B)$.)

A generalized Schrödinger operator is of the form

$$
H=W+V \text { on } \mathcal{H}^{(n)},
$$

with

$$
W=\sqrt{\mathcal{K}}(\mathbf{D}-\mathcal{A})^{*} \mathcal{R}(\mathbf{D}-\mathcal{A}) \sqrt{\mathcal{K}}
$$

Received by the editors July 18, 2001 and, in revised form, October 22, 2001.

1991 Mathematics Subject Classification. Primary 81Q10, 47F05; Secondary 35P05.

Key words and phrases. Schrödinger operator, magnetic Schrödinger operator, classical wave operator, acoustic operator, Maxwell operator, Combes-Thomas estimate.

The second author was supported in part by NSF Grants DMS-9800883 and DMS-9800860. 
where:

- $\mathbf{D}$ is a first order constant coefficient partial differential operator from $\mathcal{H}^{(n)}$ to $\mathcal{H}^{(m)}$; it is given by multiplication by an $m \times n$ matrix $D(-i \nabla)$, where, for a $d$-component vector $k, D(k)$ is an $m \times n$ matrix with entries of the form $D(k)_{r, s}=a_{r, s} \cdot k$ with $a_{r, s} \in \mathbb{C}^{d}$, for $r=1, \ldots, m, s=1, \ldots, n$. We set

$$
D_{+}=\sup \left\{\|D(k)\| ; \quad k \in \mathbb{C}^{d},|k|=1\right\}<\infty ;
$$

note $\|D(k)\| \leq D_{+}|k|$ for all $k \in \mathbb{C}^{d}$.

- $\mathcal{A}$ is the operator from $\mathcal{H}^{(n)}$ to $\mathcal{H}^{(m)}$ given by multiplication by the $m \times n$ matrix-valued measurable function $\mathcal{A}(x)$. We require $\mathcal{A}(x)$ to be locally in $L^{2}$.

- $\mathcal{K}$ and $\mathcal{R}$ are coefficients operators on $\mathcal{H}^{(n)}$ and $\mathcal{H}^{(m)}$, respectively. A coefficient operator $\mathcal{S}$ on $\mathcal{H}^{(k)}$ is a bounded, invertible operator given by multiplication by a coefficient matrix: an $k \times k$ matrix-valued measurable function $\mathcal{S}(x)$ on $\mathbb{R}^{d}$, satisfying

$$
S_{-} I_{k} \leq \mathcal{S}(x) \leq S_{+} I_{k} \text { with } 0<S_{-} \leq S_{+}<\infty
$$

( $I_{k}$ is the $k \times k$ identity matrix.)

- $V$ is a potential, an operator given by multiplication by a real-valued measurable function $V(x)$. We write $V=V^{+}-V^{-}$, with $V^{ \pm} \geq 0$, and require $V^{+}$ to be locally in $L^{1}$ and $V^{-}$to be $W$-form bounded with relative bound $<1$, i.e., there exist constants $0 \leq \Theta_{1}, \Theta_{2}<\infty$, with $\Theta_{1}<1$, such that

$$
\left\langle\psi, V^{-} \psi\right\rangle \leq \Theta_{1}\langle\psi, W \psi\rangle+\Theta_{2}\|\psi\|^{2} \text { for all } \psi \in \mathcal{Q}(W) .
$$

A generalized Schrödinger operator is well defined as a semibounded self-adjoint operator. To see this, note that $\mathbf{D}-\mathcal{A}$, initially defined on infinitely differentiable functions with compact support from $\mathcal{H}^{(n)}$ to $\mathcal{H}^{(m)}$, extends to a closed, densely defined operator. We set (as in [13, 15])

$$
A=\sqrt{\mathcal{R}}(\mathbf{D}-\mathcal{A}) \sqrt{\mathcal{K}} \text { on } \mathcal{D}(A)=\frac{1}{\sqrt{\mathcal{K}}} \mathcal{D}(\mathbf{D}-\mathcal{A}),
$$

a closed, densely defined operator from $\mathcal{H}^{(n)}$ to $\mathcal{H}^{(m)}$, and define $W$ by

$$
W=A^{*} A,
$$

a positive self-adjoint operator with $\mathcal{Q}(W)=\mathcal{D}(A)$. It follows from the conditions on $V^{ \pm}$that $H=W+V$ is a semibounded self-adjoint operator on $\mathcal{H}^{(n)}$, with

$$
H \geq\left(1-\Theta_{1}\right) W-\Theta_{2} \text { and } \mathcal{Q}(H)=\mathcal{Q}(W) \cap \mathcal{Q}\left(V^{+}\right) .
$$

Our estimates will be stated in terms of the constants $\Theta_{1}, \Theta_{2}$, and

$$
\Xi=D_{+} \sqrt{R_{+} K_{+}} .
$$

Note that while $\Xi$ depends only on $W, \Theta_{1}$ and $\Theta_{2}$ depend on both $W$ and $V$.

Examples include the Schrödinger operator (e.g., [16])

$$
H=-\Delta+V \text { on } \mathrm{L}^{2}\left(\mathbb{R}^{d}, \mathrm{~d} x\right), \text { where } \Xi=1,
$$

where $\Delta$ is the $d$-dimensional Laplacian operator, the magnetic field Schrödinger operator

$$
H=(-i \nabla-\mathbf{a}) \cdot(-i \nabla-\mathbf{a})+V \text { on } \mathrm{L}^{2}\left(\mathbb{R}^{d}, \mathrm{~d} x\right), \text { where } \Xi=1,
$$


where $\mathbf{a}$ is a vector potential, an operator from $\mathcal{H}^{(1)}$ to $\mathcal{H}^{(d)}$ given by multiplication by the vector-valued function $\mathbf{a}(x) \in \mathrm{L}_{\text {loc }}^{2}\left(\mathbb{R}^{d}, \mathrm{~d} x ; \mathbb{R}^{d}\right)$, and the classical wave operator $W$ given by (2) with $\mathcal{A}=0$ (see [13, 15]). Examples of classical wave operators include the acoustic operator

$$
W=-\frac{1}{\sqrt{\kappa}} \nabla \cdot \frac{1}{\rho} \nabla \frac{1}{\sqrt{\kappa}} \text { on } \mathrm{L}^{2}\left(\mathbb{R}^{d}, \mathrm{~d} x\right)
$$

where $\nabla$ is the $d$-dimensional gradient, $\kappa(x)$ is the compressibility, and $\varrho(x)$ is the mass density, and the Maxwell operator

$$
W=\frac{1}{\sqrt{\mu}} \nabla \times \frac{1}{\varepsilon} \nabla \times \frac{1}{\sqrt{\mu}} \text { on } \mathrm{L}^{2}\left(\mathbb{R}^{3}, \mathrm{~d} x ; \mathbb{C}^{3}\right),
$$

where $\nabla \times$ is the curl, $\mu(x)$ is the magnetic permeability, and $\varepsilon(x)$ is the dielectric constant.

Definition 1. The operator kernel of a bounded operator $B$ on $\mathcal{H}^{(n)}$ is defined by

$$
K_{B}(x, y)=\chi_{x} B \chi_{y} \text { for all } x, y \in \mathbb{R}^{d},
$$

where $\chi_{x}$ denotes the operator given by multiplication by the characteristic function of a cube of side 1 centered at $x$.

A bounded operator $B$ may or may not have an integral kernel, but it always has an operator kernel. (The choice of a cube of side 1 is arbitrary; one may fix a scale $L$ and replace the characteristic functions in (14) by characteristic functions of cubes of side $L$.) Note that $\left\|K_{B}(x, y)\right\| \leq\|B\|$ and

$$
(B \psi)_{x}=\sum_{y \in x+\mathbb{Z}^{d}} K_{B}(x, y) \psi_{y} \text { in } \mathrm{L}^{2}\left(\mathbb{R}^{d}, \mathrm{~d} x ; \mathbb{C}^{n}\right),
$$

for all $\psi \in \mathrm{L}^{2}\left(\mathbb{R}^{d}, \mathrm{~d} x ; \mathbb{C}^{n}\right)$, where $\phi_{x}=\chi_{x} \phi$. Operator kernels avoid many technical problems associated with integral kernels; they have proved very useful in the study of Anderson localization of random Schrödinger operators and classical wave operators [2, 7, 8, 9, 13, 14].

Schrödinger operators and classical wave operators satisfy a resolvent decay estimate, commonly called the Combes-Thomas estimate in the mathematical physics literature. The proofs are based on an argument of Combes and Thomas [3]. (For Schrödinger operators see [16, 1]; for classical wave operators see [7, 8, 13].) In the following theorem we state and prove a Combes-Thomas estimate for generalized Schrödinger operators, using ideas from [7, Lemma 12] and [13, Theorem 3.5]. Our proof does not use analyticity. We give explicit estimates on the constants, valid for all energies $z$. This will be of importance in Theorem 2,

Theorem 1. Let $H$ be a generalized Schrödinger operator, let $R(z)=(H-z)^{-1}$, $z \notin \sigma(H)$, be its resolvent, and let $K_{n, z}(x, y)$ be the operator kernel of $R(z)^{n}$, $n=1,2, \ldots$. Then for any $0<\gamma<1$ we have

$$
\left\|K_{n, z}(x, y)\right\| \leq\left(\left(\frac{1+\gamma}{1-\gamma}\right)^{2} \frac{1}{\eta_{z}}\right)^{n} \mathrm{e}^{\gamma m_{z} \sqrt{d}} \mathrm{e}^{-\gamma m_{z}|x-y|}
$$

for all $x, y \in \mathbb{R}^{d}$, where $\eta_{z}=\operatorname{dist}(z, \sigma(H))$ and

$$
m_{z}=\Gamma \frac{\eta_{z}}{\sqrt{\Theta_{2}+\eta_{z}+|z|}},
$$


with

$$
\Gamma=\frac{3 \sqrt{7} \sqrt{1-\Theta_{1}}}{32 \Xi} .
$$

If $\operatorname{Re} z<\Sigma=\inf \sigma(H)$ we also have

$$
\left\|K_{n, z}(x, y)\right\| \leq\left(\frac{1}{\left(1-\gamma^{2}\right)(\Sigma-R e z)}\right)^{n} \mathrm{e}^{\gamma \frac{\sqrt{\Sigma-R e z} \sqrt{d}}{\Xi}} \mathrm{e}^{-\gamma \frac{\sqrt{\Sigma-R e z}}{\Xi}}|x-y|
$$

for all $x, y \in \mathbb{R}^{d}$.

Theorem 1 is the basic tool in our derivation of estimates on operator kernels of functions of generalized Schrödinger operators. The proof is given in Section 2 ,

If the energy $z$ is real and sits in a spectral gap of the operator $H$, one can obtain estimates where the rate of the exponential decay is proportional to the product of the square roots of the distances from $z$ to the edges of the gap, similar to (19); see 1., 13].

Note that $m_{z}$ grows as $\sqrt{\eta_{z}}$ as $\eta_{z} \rightarrow \infty$, so the the constant in (16) grows as $\mathrm{e}^{c \sqrt{\eta_{z}}}$. This can be avoided by picking $\gamma=\left(2+\sqrt{\eta_{z}}\right)^{-1}$. The resulting estimate, which will be used in the proof of Theorem 2 is given in the following corollary.

Corollary 1. Let $H$ be a generalized Schrödinger operator. Then, with the notation of Theorem 1 ,

$$
\left\|K_{n, z}(x, y)\right\| \leq\left(\frac{9}{\eta_{z}}\right)^{n} \mathrm{e}^{\Gamma \sqrt{d}} \mathrm{e}^{-\frac{m_{z}}{2+\sqrt{\eta} z}|x-y|}
$$

for all $x, y \in \mathbb{R}^{d}$.

If $f \in C_{0}(\mathbb{R})$ (the continuous functions vanishing at infinity), it follows from the Stone-Weierstrass theorem and Corollary 1 that the operator kernel $K_{f}(x, y)$ of $f(H)$ goes to 0 as $|x-y| \rightarrow \infty$. But this soft argument gives no estimate on the rate of decay. In fact, it is known that for $H=-\Delta$ the kernel of $f(H)$ will not have rapid decay as $|x-y| \rightarrow \infty$ if $f$ is not smooth [16, Remark 3 on p. 476].

In this article we combine Corollary 1 with the Helffer-Sjöstrand formula [11] to derive estimates on the decay of operator kernels of functions of generalized Schrödinger operators. The existing literature derives estimates on integral kernels from the heat kernel estimate [16, 4, 5. But heat kernels estimates are only available for Schrödinger operators [16] and acoustic operators with constant compressibility [5]; they are not available for Maxwell operators, for example. We show that a Combes-Thomas estimate as in Corollary 1 is a natural tool to obtain kernel estimates for a large class of operators. Even for Schrödinger operators polynomial decay for the (integral or operator) kernel of $f(H)$ with $f$ an arbitrary function in $C_{0}^{\infty}(\mathbb{R})$ is an open question (see [16, p. 476]). In the case when there is a heat kernel estimate, Davies [5, Theorem 3.4.10] proved polynomial decay of any order for $f \in \mathcal{S}(\mathbb{R})$. In Theorem 2 we allow any generalized Schrödinger operator and prove polynomial decay of any order if $f$ belongs to the much larger class of slowly decreasing smooth functions (see Definition 21). A particular example of interest [10] is $f(u)=\frac{1-\mathcal{X}_{I}(u)}{u-E}$, where $I \subset \sigma(H)$ is a bounded open interval, $E \in I$, and $\mathcal{X}_{I}$ is a smooth characteristic function for the interval $I$. Note that in this case we have $f(H)=\left(1-\mathcal{X}_{I}(H)\right) R(E)$ with $E \in \sigma(H)$.

If $w \in \mathbb{R}^{n}$ we write

$$
\langle w\rangle=\sqrt{1+|w|^{2}}
$$


Definition 2. $f: \mathbb{R} \rightarrow \mathbb{C}$ is called a slowly decreasing smooth function if it is infinitely differentiable and there exists $\gamma>0$ such that

$$
\left|f^{(n)}(u)\right| \leq \frac{c_{n}}{\langle u\rangle^{\gamma+n}}
$$

for some constants $c_{n}<\infty$, all $u \in \mathbb{R}$, and all $n=0,1,2, \ldots$

If $f$ is a slowly decreasing smooth function, we define the norms [6] eq. (2.1.3)]:

$$
\|f\|_{n}=\sum_{r=0}^{n} \int_{\mathbb{R}}\left|f^{(r)}(u)\right|\langle u\rangle^{r-1} \mathrm{~d} u, \quad n=1,2, \ldots .
$$

Theorem 2. Let $H$ be a generalized Schrödinger operator and let $f$ be a slowly decreasing smooth function. Then the operator kernel $K_{f}(x, y)$ of $f(H)$ decays faster than any polynomial as $|x-y| \rightarrow \infty$. More precisely, there is a finite constant $\widetilde{C}$, independent of $f$ and $H$, such that for all $k=1,2, \ldots$ we have

$$
\left\|K_{f}(x, y)\right\| \leq \widetilde{C} \mathrm{e}^{\Gamma \sqrt{d}}\|f\|_{k+2} \frac{\left\langle 3 k\left(\Theta_{2}+8\right) \Gamma^{-1}\right\rangle^{k}}{\langle x-y\rangle^{k}}
$$

for all $x, y \in \mathbb{R}^{d}$.

Theorem 2 is proved in Section 3. The proof is based on the estimate (20) and the Helffer-Sjöstrand formula. Theorem 2 provides a crucial ingredient for the results in [10.

\section{The Combes-Thomas estimate}

In this section we prove Theorem 1. We start by defining the operators formally given by

$$
H_{\alpha}=\mathrm{e}^{\alpha \cdot x} H \mathrm{e}^{-\alpha \cdot x} \text { and } W_{\alpha}=\mathrm{e}^{\alpha \cdot x} W \mathrm{e}^{-\alpha \cdot x} \text {, where } \alpha \in \mathbb{R}^{d} ;
$$

note that

$$
H_{\alpha}=W_{\alpha}+V .
$$

To do so, let us consider the bounded operator $G_{\alpha}$, defined as

$$
G_{\alpha}=\sqrt{\mathcal{R}} D(\alpha) \sqrt{\mathcal{K}}, \text { so }\left\|G_{\alpha}\right\| \leq|\alpha| \Xi,
$$

as in [13, Theorem 3.5]. Then

$$
\begin{aligned}
A_{\alpha} & =\mathrm{e}^{\alpha \cdot x} A \mathrm{e}^{-\alpha \cdot x}=A+i G_{\alpha} \text { on } \mathcal{D}(A), \\
\left(A^{*}\right)_{\alpha} & =\mathrm{e}^{\alpha \cdot x} A^{*} \mathrm{e}^{-\alpha \cdot x}=A^{*}+i G_{\alpha}^{*} \text { on } \mathcal{D}\left(A^{*}\right),
\end{aligned}
$$

are closed, densely defined operators. (Note $\left(A^{*}\right)_{\alpha} \neq\left(A_{\alpha}\right)^{*}$.) We now define $W_{\alpha}=\left(A^{*}\right)_{\alpha} A_{\alpha}$ as a quadratric form with form domain $\mathcal{D}(A)=\mathcal{Q}(W)$, i.e., for each $\alpha \in \mathbb{R}^{d}$,

$$
W_{\alpha}[\psi]=\left\langle\left(A^{*}\right)_{\alpha}^{*} \psi, A_{\alpha} \psi\right\rangle, \quad \psi \in \mathcal{D}(A) .
$$

If $\alpha=0, W_{0}$ is the closed, nonegative quadratic form associated to the operator $W$. We also define the quadratic form $H_{\alpha}=W_{\alpha}+V$ on $\mathcal{Q}(W) \cap \mathcal{Q}\left(V^{+}\right)$.

It follows from (27) and (28) that

$$
H_{\alpha}[\psi]-H[\psi]=W_{\alpha}[\psi]-W[\psi]=2 i \operatorname{Re}\left\langle A \psi, G_{\alpha} \psi\right\rangle-\left\langle G_{\alpha} \psi, G_{\alpha} \psi\right\rangle,
$$


so, using (26) and (8), we get

$$
\begin{aligned}
\mid H_{\alpha} & {[\psi]-H[\psi] \mid=\left(4\left(\operatorname{Re}\left\langle A \psi, G_{\alpha} \psi\right\rangle\right)^{2}+\left\|G_{\alpha} \psi\right\|^{4}\right)^{\frac{1}{2}} } \\
& \leq\left\|G_{\alpha} \psi\right\|\left(4\|A \psi\|^{2}+\left\|G_{\alpha} \psi\right\|^{2}\right)^{\frac{1}{2}} \leq 2 s W[\psi]+\frac{1}{2}\left(\frac{1}{s}+s\right)|\alpha|^{2} \Xi^{2}\|\psi\|^{2} \\
& \leq \frac{2 s}{1-\Theta_{1}} H[\psi]+\left(\frac{2 s \Theta_{2}}{1-\Theta_{1}}+\frac{1}{2}\left(\frac{1}{s}+s\right)|\alpha|^{2} \Xi^{2}\right)\|\psi\|^{2}
\end{aligned}
$$

for any $s>0$. It follows [12, Theorem VI.1.33] that $H_{\alpha}$ is a closed sectorial form on the form domain of $H$. We define $H_{\alpha}$ as the unique $m$-sectorial operator associated with it [12, Theorem VI.2.1].

Let $z \notin \sigma(H)$, if $0<\gamma<1$ and

$$
\Omega=2\|(a+b H) R(z)\| \leq \gamma
$$

where

$$
a=\frac{2 s \Theta_{2}}{1-\Theta_{1}}+\frac{1}{2}\left(\frac{1}{s}+s\right)|\alpha|^{2} \Xi^{2}, \quad b=\frac{2 s}{1-\Theta_{1}} .
$$

It follows from [12, Theorem VI.3.9] that $z \notin \sigma\left(H_{\alpha}\right)$ and

$$
\left\|R(z)-R_{\alpha}(z)\right\| \leq \frac{4 \Omega}{(1-\Omega)^{2}}\|R(z)\| \leq \frac{4 \gamma}{(1-\gamma)^{2} \eta_{z}},
$$

where $R_{\alpha}(z)=\left(H_{\alpha}-z\right)^{-1}$ and $\eta_{z}=\operatorname{dist}(z, \sigma(H))$, so

$$
\left\|R_{\alpha}(z)\right\| \leq\left(\frac{1+\gamma}{1-\gamma}\right)^{2} \frac{1}{\eta_{z}}
$$

Since

$$
\Omega \leq \frac{2}{\eta_{z}}\left(a+b\left(\eta_{z}+|z|\right)\right)
$$

(32) will be satisfied if

$$
A s+\left(\frac{1}{s}+s\right)|\alpha|^{2} \leq B
$$

with

$$
A=\frac{4}{\left(1-\Theta_{1}\right) \Xi_{2}^{2}}\left(\Theta_{2}+\eta_{z}+|z|\right), \quad B=\frac{\gamma \eta_{z}}{\Xi^{2}} .
$$

The left hand side of (137) is minimized by the choice

$$
s=\frac{|\alpha|}{\sqrt{A+|\alpha|^{2}}}
$$

in which case (37) becomes

$$
2|\alpha| \sqrt{A+|\alpha|^{2}} \leq B
$$

i.e.,

$$
4|\alpha|^{4}+4 A|\alpha|^{2}-B^{2} \leq 0,
$$

so it suffices to require

$$
|\alpha|^{2} \leq \frac{1}{2}\left(-A+\sqrt{A^{2}+B^{2}}\right)=\frac{A}{2}\left(\sqrt{1+\frac{B^{2}}{A^{2}}}-1\right) \equiv \beta .
$$


Since $\sqrt{1+x} \geq 1+\frac{x}{2}-\frac{x^{2}}{8}$ for $x \geq 0$, we have

$$
\begin{aligned}
\beta & \geq \frac{B^{2}}{4 A}\left(1-\frac{B^{2}}{4 A^{2}}\right) \geq \frac{B^{2}}{4 A}\left(1-\frac{\gamma^{2}\left(1-\Theta_{1}\right)^{2}}{64}\right) \\
& \geq \frac{63 B^{2}}{256 A}=\frac{63\left(1-\Theta_{1}\right) \gamma^{2} \eta_{z}^{2}}{1024 \Xi^{2}\left(\Theta_{2}+\eta_{z}+|z|\right)}=\gamma^{2} m_{z}^{2},
\end{aligned}
$$

with $m_{z}$ as in (17).

Thus, if we take $|\alpha| \leq \gamma m_{z}$, we satisfy (32) and obtain (35).

Now let $x_{0}, y_{0} \in \mathbb{R}^{d}, n \in \mathbb{N}$, and take $\alpha=\gamma \frac{m_{z}}{\left|x_{0}-y_{0}\right|}\left(x_{0}-y_{0}\right)$. We have

$$
\begin{aligned}
& K_{n, z}\left(x_{0}, y_{0}\right)=\chi_{x_{0}} R(z)^{n} \chi_{y_{0}}=\chi_{x_{0}} \mathrm{e}^{-\alpha \cdot x} R_{\alpha}(z)^{n} \mathrm{e}^{\alpha \cdot x} \chi_{y_{0}} \\
& \quad=\mathrm{e}^{-\gamma m_{z}\left|x_{0}-y_{0}\right|} \chi_{x_{0}} \mathrm{e}^{-\alpha \cdot\left(x-x_{0}\right)} R_{\alpha}(z)^{n} \mathrm{e}^{\alpha \cdot\left(x-y_{0}\right)} \chi_{y_{0}},
\end{aligned}
$$

SO

$$
\begin{aligned}
& \left\|K_{n, z}\left(x_{0}, y_{0}\right)\right\| \\
& \quad \leq\left(\left(\frac{1+\gamma}{1-\gamma}\right)^{2} \frac{1}{\eta_{z}}\right)^{n}\left\|\chi_{x_{0}} \mathrm{e}^{-\alpha \cdot\left(x-x_{0}\right)}\right\|_{\infty}\left\|\chi_{y_{0}} \mathrm{e}^{\alpha \cdot\left(x-y_{0}\right)}\right\|_{\infty} \mathrm{e}^{-\gamma m_{z}\left|x_{0}-y_{0}\right|} .
\end{aligned}
$$

Since

$$
\left\|\chi_{x_{0}} \mathrm{e}^{ \pm \alpha \cdot\left(x-x_{0}\right)}\right\|_{\infty} \leq \mathrm{e}^{\frac{\sqrt{d}}{2}|\alpha|}=\mathrm{e}^{\frac{\sqrt{d}}{2} \gamma m_{z}}
$$

(16) follows.

Now let $\operatorname{Re} z<\Sigma=\inf \sigma(H)$. It follows from (30) and (26) that

$$
\operatorname{Re} H_{\alpha}[\psi]=H[\psi]-\left\langle G_{\alpha} \psi, G_{\alpha} \psi\right\rangle \geq \Sigma-|\alpha|^{2} \Xi^{2} .
$$

As $H_{\alpha}$ is an $m$-sectorial operator, it follows that $z \notin \sigma\left(H_{\alpha}\right)$ as long as we have $|\alpha|^{2} \Xi^{2}<\Sigma-\operatorname{Re} z$ (see [12, p. 279]). Moreover, if $0<\gamma<1$ and

$$
|\alpha| \leq \gamma \frac{\sqrt{\Sigma-R e z}}{\Xi}
$$

we have

$$
\left\|R_{\alpha}(z)\right\| \leq \frac{1}{\left(1-\gamma^{2}\right)(\Sigma-R e z)} .
$$

Proceeding as in (44), we conclude that (19) follows from (48) and (49)).

Theorem 1 is proven.

\section{KeRnel eStimates FOR SLOWLY DECREASING SMOOTH FUnCTIONS}

In this section we prove Theorem 2 Let $f$ be a slowly decreasing smooth function satisfying (21). We recall the Helffer-Sjöstrand formula 11] (see also 66. Section $2.2])$ :

$$
f(H)=\frac{1}{\pi} \int_{\mathbb{R}^{2}} \frac{\partial \tilde{f}_{n}(z)}{\partial \bar{z}} R(z) \mathrm{d} u \mathrm{~d} v,
$$

where $z=u+i v, n=1,2, \ldots$, and

$$
\tilde{f}_{n}(z)=\left\{\sum_{r=0}^{n} \frac{1}{r !} f^{(r)}(u)(i v)^{r}\right\} \sigma(u, v),
$$


called a quasi-analytic extension of $f$ of order $n$, with $\sigma(u, v)=\tau\left(\frac{v}{\langle u\rangle}\right)$ for some infinitely differentiable function $\tau(s)$ on the real line, which equals 1 if $|s|<1$ and equals 0 if $|s|>2$. We have

$$
\begin{aligned}
\frac{\partial \tilde{f}_{n}(z)}{\partial \bar{z}}= & \frac{1}{2}\left\{\frac{\partial \tilde{f}_{n}(z)}{\partial u}+i \frac{\partial \tilde{f}_{n}(z)}{\partial v}\right\} \\
= & \frac{1}{2}\left\{\sum_{r=0}^{n} \frac{1}{r !} f^{(r)}(u)(i v)^{r}\right\}\left\{\frac{\partial \sigma(u, v)}{\partial u}+i \frac{\partial \sigma(u, v)}{\partial v}\right\} \\
& +\frac{1}{2 n !} f^{(n+1)}(u)(i v)^{n} \sigma(u, v) .
\end{aligned}
$$

The integral in (50) converges in operator norm, The identity (50) holds for any $n=1,2, \ldots$ and any choice of the function $\tau(s)$ (we choose $\tau(s)$ once for all, with $0 \leq \tau(s) \leq 1)$. We also have the following bound [6, p. 25]:

$$
\begin{aligned}
& \left|\frac{\partial \tilde{f}_{n}(z)}{\partial \bar{z}}\right| \\
& \quad \leq C\left\{\sum_{r=0}^{n} \frac{1}{r !}\left|f^{(r)}(u)\right| \frac{|v|^{r}}{\langle u\rangle}\right\} \chi_{A}(u, v)+\frac{1}{2 n !}\left|f^{(n+1)}(u)\right||v|^{n} \chi_{B}(u, v)
\end{aligned}
$$

where $A=\{\langle u\rangle<|v|<2\langle u\rangle\}, B=\{0<|v|<2\langle u\rangle\}, \chi_{A}$ and $\chi_{B}$ are the corresponding characteristic functions, and $C$ is some constant depending only on our choice of $\tau(s)$. Thus there is a finite constant $c$, independent of $f$ and $n$, such that [6, Lemma 2.2.1]

$$
\|f(H)\| \leq c\|f\|_{n+1} \text { for all } n=1,2, \ldots .
$$

It follows that the operator kernel $K_{f}(x, y)$ of $f(H)$ is given by

$$
K_{f}(x, y)=\frac{1}{\pi} \int_{\mathbb{R}^{2}} \frac{\partial \tilde{f}_{n}(z)}{\partial \bar{z}} K_{z}(x, y) \mathrm{d} u \mathrm{~d} v
$$

for any $n=1,2, \ldots$, where $K_{z}(x, y)=K_{1, z}(x, y)$ is the operator kernel of the resolvent $R(z)$. To estimate $\left\|K_{f}(x, y)\right\|$ we use (53) and (20), and make use in (56) and (57) of the fact that the function $g(s)=\frac{s}{(a+\sqrt{s}) \sqrt{b+s}}$, with $a, b \geq 0$, is increasing for $s \geq 0$. Note that $\eta_{z} \geq|v|$ and hence

$$
\frac{m_{z}}{2+\sqrt{\eta_{z}}} \geq \frac{\Gamma|v|}{(2+\sqrt{|v|}) \sqrt{\Theta_{2}+|v|+|z|}} .
$$

Thus, if $(u, v) \in A$, we have $|v| \geq 1$ and $|z| \leq \sqrt{2}|v|$, hence

$$
\frac{m_{z}}{2+\sqrt{\eta_{z}}} \geq \frac{\Gamma}{3 \sqrt{\Theta_{2}+1+\sqrt{2}}} \geq \frac{\Gamma}{3 \sqrt{\Theta_{2}+3}}
$$


so for any $r=0,1,2, \ldots$ it follows that

$$
\begin{aligned}
\int_{\mathbb{R}^{2}} & \left|f^{(r)}(u)\right| \frac{|v|^{r}}{\langle u\rangle} \chi_{A}(u, v)\left\|K_{z}(x, y)\right\| \mathrm{d} u \mathrm{~d} v \\
\leq & 9 \mathrm{e}^{\Gamma \sqrt{d}}\left[\int_{\mathbb{R}^{2}}\left|f^{(r)}(u)\right| \frac{|v|^{r-1}}{\langle u\rangle} \chi_{A}(u, v) \mathrm{d} u \mathrm{~d} v\right] \mathrm{e}^{-\frac{\Gamma}{3 \sqrt{\Theta_{2}+3}}|x-y|} \\
\leq & 9 \mathrm{e}^{\Gamma \sqrt{d}}\left[t_{r} \int_{\mathbb{R}}\left|f^{(r)}(u)\right|\langle u\rangle^{r-1} \mathrm{~d} u\right] \mathrm{e}^{-\frac{\Gamma}{\sqrt{\Theta_{2}+3}}|x-y|}
\end{aligned}
$$

where $t_{0}=2 \log 2, t_{r}=\frac{2^{r+1}}{r}$ for $r=1,2 \ldots, n$. We can thus conclude that

$$
\begin{array}{r}
C \int_{\mathbb{R}^{2}}\left\{\sum_{r=0}^{n} \frac{1}{r !}\left|f^{(r)}(u)\right| \frac{|v|^{r}}{\langle u\rangle}\right\} \chi_{A}(u, v)\left\|K_{z}(x, y)\right\| \mathrm{d} u \mathrm{~d} v \\
\leq \widehat{C}_{1} \mathrm{e}^{\Gamma \sqrt{d}}\|f\|_{n} \mathrm{e}^{-\frac{\Gamma}{3 \sqrt{\Theta_{2}+3}}|x-y|},
\end{array}
$$

where $\widehat{C}_{1}=C \max _{r} \frac{t_{r}}{r !}<\infty$.

When $(u, v) \in B$, we have $|v| \leq 2\langle u\rangle$ and $|z| \leq \sqrt{5}\langle u\rangle \leq 3\langle u\rangle$. Using these inequalities in (56), we get (using $2 a b \leq a^{2}+b^{2}$ in the third inequality)

$$
\begin{aligned}
\frac{m_{z}}{2+\sqrt{\eta_{z}}} & \geq \frac{\Gamma|v|}{(2+\sqrt{2\langle u\rangle}) \sqrt{\Theta_{2}+5\langle u\rangle}} \geq \frac{\Gamma|v|}{2 \sqrt{(2+\langle u\rangle)\left(\Theta_{2}+5\langle u\rangle\right)}} \\
& \geq \frac{\Gamma|v|}{\Theta_{2}+2+6\langle u\rangle} \geq \frac{\Gamma|v|}{\left(\Theta_{2}+8\right)\langle u\rangle} .
\end{aligned}
$$

If $k$ is a positive integer, we recall that for all $t \geq 0$ we have

$$
\mathrm{e}^{-t} \leq \frac{b_{k}}{t^{k}}, \text { where } b_{k}=\mathrm{e}^{-k} k^{k} .
$$

Thus, if $n \geq k+1$, we have

$$
\begin{aligned}
\frac{1}{2 n !} & \int_{\mathbb{R}^{2}}\left|f^{(n+1)}(u)\left\|\left.v\right|^{n} \chi_{B}(u, v)\right\| K_{z}(x, y) \| \mathrm{d} u \mathrm{~d} v\right. \\
& \leq \frac{9 \mathrm{e}^{\Gamma \sqrt{d}}\left(\Theta_{2}+8\right)^{k} b_{k}}{2 n ! \Gamma^{k}|x-y|^{k}} \int_{\mathbb{R}^{2}}\left|f^{(n+1)}(u) \| v\right|^{n-k-1}\langle u\rangle^{k} \chi_{B}(u, v) \mathrm{d} u \mathrm{~d} v \\
& \leq \frac{9 \mathrm{e}^{\Gamma \sqrt{d}}\left(\Theta_{2}+8\right)^{k} b_{k}}{n ! \Gamma^{k}|x-y|^{k}} \frac{2^{n-k}}{n-k} \int_{\mathbb{R}}\left|f^{(n+1)}(u)\right|\langle u\rangle^{n} \mathrm{~d} u .
\end{aligned}
$$

If we now pick $n=k+1$, it follows that for any $k=1,2, \ldots$ we have

$$
\begin{gathered}
\frac{1}{2(k+1) !} \int_{\mathbb{R}^{2}}\left|f^{(k+2)}(u)\left\|\left.v\right|^{k+1} \chi_{B}(u, v)\right\| K_{z}(x, y) \| \mathrm{d} u \mathrm{~d} v\right. \\
\leq \widehat{C}_{2} \frac{\mathrm{e}^{\Gamma \sqrt{d}}\left(\Theta_{2}+8\right)^{k}}{\Gamma^{k}|x-y|^{k}} \int_{\mathbb{R}}\left|f^{(k+2)}(u)\right|\langle u\rangle^{k+1} \mathrm{~d} u,
\end{gathered}
$$

with $\widehat{C}_{2}=18 \max _{k} \frac{b_{k}}{(k+1) !}<\infty$.

It now follows from (55), (53), (59), (63), 22), and (61) that we have

$$
\begin{aligned}
\left\|K_{f}(x, y)\right\| & \leq \widetilde{C} \mathrm{e}^{\Gamma \sqrt{d}}\|f\|_{k+2}\left(\mathrm{e}^{-\frac{\Gamma}{3 \sqrt{\Theta_{2}+3}}|x-y|}+\frac{\left(\Theta_{2}+8\right)^{k}}{\Gamma^{k}|x-y|^{k}}\right) \\
& \leq 2 \widetilde{C} \mathrm{e}^{\Gamma \sqrt{d}}\|f\|_{k+2} \frac{\left(3 k\left(\Theta_{2}+8\right) \Gamma^{-1}\right)^{k}}{|x-y|^{k}}
\end{aligned}
$$


for any $k=1,2, \ldots$, where $\widetilde{C}<\infty$ is some constant independent of $k, f$, and $H$. Since we have $\left\|K_{f}(x, y)\right\| \leq c\|f\|_{k+2}$ by (54), and $\min \left\{1,\left|\frac{a}{b}\right|\right\} \leq \frac{\langle a\rangle}{\langle b\rangle}$, the estimate (23) follows with some different constant $\widetilde{C}$.

Theorem 2 is proven.

\section{ACKNOWLEDGEMENT}

The authors thank Vojkan Jaksic for calling the Helffer-Sjöstrand formula to their attention.

\section{REFERENCES}

[1] Barbaroux, J.M., Combes, J.M., Hislop, P.D.: Localization near band edges for random Schrödinger operators. Helv. Phys. Acta 70, 16-43 (1997) MR 98h:82028

[2] Combes, J.M., Hislop, P.D.: Localization for some continuous, random Hamiltonian in ddimension. J. Funct. Anal. 124, 149-180 (1994) MR 95g:82047

[3] Combes, J.M., Thomas, L.: Asymptotic behavior of eigenfunctions for multi-particle Schrödinger operators. Commun. Math. Phys. 34, 251-270 (1973) MR 52:12611

[4] Davies, E.B.: Kernel estimates for functions of second order elliptic operators. Quart. J. Math. Oxford (2) 39, 37-46 (1988) MR 89c:35123

[5] Davies, E.B.: Heat kernels and spectral theory. Cambridge University Press, 1989 MR 90e:35123

[6] Davies, E.B.: Spectral theory and differential operators. Cambridge University Press, 1995 MR 96h:47056

[7] Figotin, A., Klein, A.: Localization of classical waves I: Acoustic waves. Commun. Math. Phys. 180, 439-482 (1996) MR 2000d:35240a

[8] Figotin, A., Klein, A.: Localization of classical waves II: Electromagnetic waves. Commun. Math. Phys. 184, 411-441 (1997) MR 2000d:35240b

[9] Germinet, F., Klein, A.: Bootstrap Multiscale Analysis and Localization in random media. Commun. Math. Phys. 222, 415-448 (2001)

[10] Germinet, F., Klein, A.: A characterization of the Anderson metal-insulator transport transition. Preprint.

[11] Helffer, B., Sjöstrand, J.: Equation de Schrödinger avec champ magnétique et équation de Harper. In Schrödinger Operators, H. Holden and A. Jensen, eds., pp. 118-197. Lecture Notes in Physics 345, Springer-Verlag, 1989 MR 91g:35078

[12] Kato, T.: Perturbation Theory for Linear Operators. Springer-Verlag, 1976 MR 53:11389

[13] Klein, A., Koines, A.: A general framework for localization of classical waves: I. Inhomogeneous media and defect eigenmodes. Math. Phys. Anal. Geom. 4, 97-130 (2001)

[14] Klein, A., Koines, A.: A general framework for localization of classical waves: II. Random media. In preparation.

[15] Klein, A., Koines, A., Seifert, M.: Generalized eigenfunctions for waves in inhomogeneous media. J. Funct. Anal. 190, 255-291 (2002)

[16] Simon, B.: Schrödinger semi-groups. Bull. Amer. Math. Soc. 7, 447-526 (1982) MR 86b:81001a

UMR 8524 CNRS, UFR de Mathématiques, Université de Lille 1, F-59655 Villeneuve D'AscQ Cédex, France

E-mail address: germinet@agat.univ-lille1.fr

Department of Mathematics, University of California, Irvine, California 92697-3875

E-mail address: aklein@uci.edu 